\title{
Chorionic Villous Mesenchymal Stem Cells Derived Exosomes Deliver miR-135b-5p to Trophoblasts, Promoting their Proliferation and Invasion by Targeting TXNIP via $\beta$-Catenin Pathway
}

\section{Yijing Chu}

The Affiliated Hospital of Qingdao University

Yan Zhang

affiliated hospital of qingdao unviersity

\section{Guoqiang Gao}

the affiliated hospital of qingdao university

Jun Zhou

the affiliated hospital of qingdao university

Yang Lv

the affiliated hospital of qingdao university

Ning Zhang

the affiliated hospital of qingdao university

Jing Li

The Affiliated Hospital of Qingdao University

Ling Liu

the affiliated hospital of qingdao university

\section{Ping Tan}

The Affiliated Hospital of Qingdao University

\section{Xiaofei Wang}

The Affiliated Hospital of Qingdao University

\section{Ke Yao}

The Affiliated Hospital of Qingdao University

Chong Liu

The Affiliated Hospital of Qingdao University

\section{Huansheng Zhou}

The Affiliated Hospital of Qingdao University

Min Li

Affiliated Hospital of Medical College Qingdao University: The Affiliated Hospital of Qingdao University

\section{Min Zhao}

The Affiliated Hospital of Qingdao University 


\section{Han Yang}

the affiliated hospital of qingdao university

Xiaoyu $\mathrm{Hu}$

the affiliated hospital of qingdao university

\section{Yan Wang}

the affiliated hospital of qingdao university

\section{Yuanhua Ye ( $D$ yeyuanhua8067@163.com )}

the affiliated hospital of qingdao university https://orcid.org/0000-0002-6460-9160

\section{Research}

Keywords: CV-MSCs, TXNIP, miR-135b-5p, $\beta$-catenin, trophoblasts

Posted Date: December 28th, 2020

DOl: https://doi.org/10.21203/rs.3.rs-133394/v1

License: (c) (1) This work is licensed under a Creative Commons Attribution 4.0 International License.

Read Full License 


\section{Abstract}

Background: Human chorionic villous mesenchymal stem cells (CV-MSCs) are found to be a promising and effective treatment for tissue injury. Trophoblast dysfunction during pregnancies is significantly involved in the pathogenesis of preeclampsia (PE). This work was to understand how CV-MSCs regulated trophoblast function.

Methods: In this study, we treated trophoblasts with CV-MSC-derived exosomes and RNA-seq analysis was used to understand the changes in trophoblasts. We examined the levels of TXNIP and $\beta$-catenin in trophoblasts by immunohistochemistry, western blot and qRT-PCR assays. Luciferase reporter assays and qRT-PCR assays were used to understand the role of miR135b-5p in the effects of CV-MSC-derived exosomes. The growth and invasion of trophoblasts was evaluated with the CCK-8 and transwell assays.

Results: The treatment markedly enhanced the trophoblast proliferation and invasion. Furthermore, a significant decrease of TXNIP expression and inactivation of the $\beta$-catenin pathway in CV-MSCs exosomes-treated trophoblasts was observed. Consistent with these findings, TXNIP inhibition exhibited the same effect of promoting trophoblast proliferation and invasion as induced by CV-MSC-derived exosomes, also with the accompaniment of inactivation of $\beta$-catenin pathway. In addition, overexpression of TXNIP activated the $\beta$-catenin pathway in trophoblasts, and reduced the proliferation and invasion of trophoblasts. Importantly, miR135b-5p was found to be highly expressed in CV-MSC exosomes and interact with TXNIP. The miR-135b-5p overexpression significantly elevated the proliferation and invasion of trophoblasts, which could be attenuated by TXNIP overexpression.

Conclusion: Our results suggest that TXNIP-dependent $\beta$-catenin pathway inactivation mediated by miR135b-5p which is delivered by CV-MSC-derived exosomes could promote the proliferation and invasion of trophoblasts.

\section{Introduction}

In humans, aberrant development of placenta could result into pregnancy complications, including preeclampsia $(\mathrm{PE})^{1}$. Trophoblast invasion into the decidua for accessing maternal blood supply is crucial for fetal development ${ }^{2}$. The placenta in PE patients is subjected to an excess of oxidative stress and inflammation, inducing anomalous differentiation of trophoblasts and a significant increase of antiangiogenic protein secretion ${ }^{3}$. PE proceeds from products released from the placenta, with inadequate perfusion and high oxidative stress, into the maternal blood, stimulating a systemic disorder in endothelia ${ }^{4}$. Furthermore, the clinical outcome of PE depends on the extent and the number of arteries invaded by the defective trophoblasts ${ }^{5}$. Since the exact etiology of PE is unclear, understanding the mechanisms in the proliferation and invasion of trophoblasts are important for PE treatment.

Mesenchymal stem cells (MSCs) feature immunomodulatory, anti-inflammatory, angiogenic, and antiapoptotic effects in tissue repairing ${ }^{6}$. Fetus or placenta-derived MSCs exhibit ontological 
characteristics that could consign more advantages, in comparison with their adult equivalents ${ }^{7,8}$. CVMSCs provide a distinctive range of mediators which can impose regulation on the main functions in other cells ${ }^{9}$. These characteristics render CV-MSCs promising candidates for cell-based treatment ${ }^{10}$. Exosomes are homogeneous membrane vesicles secreted by various cell types which confer different attributes on exosomes ${ }^{11}$. Exosomes carry complicated and characteristic components, including proteins, lipids, mRNAs, and miRNAs ${ }^{12}$. Several studies reveal that exosomes participate in the cellular communication, immune response, antigen presentation as well as cellular migration/differentiation, affecting processes both in physiology and pathology ${ }^{13}$. miRNAs are a fragment of endogenous noncoding RNAs which are responsible for various cellular regulations ${ }^{14}$. Multiple miRNA expressions were observed in human placentas ${ }^{15}$ and abnormality of miRNA expression was perceived in the placentas of patients with pregnancy complications, including $\mathrm{PE}^{16}$. Changes in the expression of various miRNAs were also demonstrated in placental trophoblasts in hypoxic conditions ${ }^{17}$.

Thioredoxin-interacting protein (TXNIP) endogenously inhibits the thioredoxin system and plays a key role in redox homeostasis ${ }^{18,19}$. It can regulate different biological processes, as cell growth and angiogenesis in nucleus, and modulation of apoptosis signal-regulating kinase-1 (ASK-1) and cell membrane receptor signaling in cytosol ${ }^{20,21}$. TXNIP elevates the level of reactive oxygen species (ROS) and contributes to oxidative stress and apoptosis ${ }^{22}$. TXNIP can also regulate redox/glucose-mediated stress and inflammation, and is reported to overexpress in ischemia-hypoxia/reoxygenation and irradiation injuries in many organs, thus providing a promising therapeutic $\operatorname{target}^{23-25}$.

In this study, we studied the effects of CV-MSC-derived exosomes on the proliferative and invasive capacities of the trophoblast cell lines, JAR and HTR-8/SVneoc. Transcriptome analyses manifested considerable downregulation of TXNIP and upregulation of WNT signalling in trophoblasts. In addition, the expression of microRNA 135b-5p was increased in trophoblasts treated with CV-MSC-derived exosomes, and affected the TXNIP expression. MiR-135b-5p putatively served as an upstream regulator of proliferation and invasion of trophoblasts through TXNIP and $\beta$-catenin regulation.

\section{Methods And Materials}

\section{Cell culture}

Placentas were acquired from pregnancies at full term through caesarean delivery with parental permission. Every procedure was performed according to ethical requirement by the Affiliated Hospital of Qingdao University, China. CV-MSCs used were between passages 3 to 6 . Briefly, the placental fetal part was sliced into roughly $1 \mathrm{~mm}^{3}$ pieces and washed by PBS. Tissue were digested by trypsin $(0.25 \%)$ and collagenase I $(0.1 \%)$ (Sigma-Aldrich, St. Louis, MO), followed by incubation at $37^{\circ} \mathrm{C}$ for half an hour. Following nylon filter filtration and centrifugation, the cells were seeded in a stem cell culture medium (SCCM), supplemented with Stem Cell Basic Medium (Dakewe Biotech Co., Guangzhou, China) and 5\% UltraGRO ${ }^{\text {TM }}$ (Helios, USA). 
JAR and HTR-8/SVneo cell lines were procured from Type Culture Collection China Centre. DMEM/F12 $(10 \% \mathrm{FBS})$ was used for cell incubation in a $5 \% \mathrm{CO}_{2}$ atmosphere at $37^{\circ} \mathrm{C}$.

\section{CV-MSC identification}

The cellular biomarkers in CV-MSCs (at passage 3) were detected by a flow cytometry using a series of antibodies (eBioscience, San Diego, CA); the biomarkers contained positive markers (CD44, CD73, CD90 and CD105) and negative markers (CD34, CD45, CD146, IG1 and HLA-DR).

Since CV-MSCs from placentas of normal pregnancies or placentas with severe PE could differentiate into osteoblasts as well as adipocytes, we assessed their differentiation capability. CV-MSCs were cultured in 6-well plates until reaching a confluency between $70 \%$ and $80 \%$. Subsequently, the CV-MSCs were transferred into osteogenic or adipogenic differentiation medium (Gibco, Carlsbad, CA) for 3 weeks of incubation. Then CV-MSCs were stained by Alizarin red S for verifying differentiation of osteoblasts, followed by oil red $\mathrm{O}$ staining for adipocyte differentiation.

\section{Exosome isolation}

Epidural CV-MSC supernatants were differentially centrifuged to obtain exosomes. The medium was disposed of after attaining 70\% confluency. DMEM/F12 (serum-free) was then adopted to culture cells for an additional day. The supernatants were pooled and sequentially centrifuged at $15,000 \mathrm{xg}$ for half an hour, followed by filtration through filters $(0.22 \mathrm{~mm})$ (Millipore, Billerica, MA) and ultracentrifuge at 120,000 x g. The exosomes were rinsed by sterile PBS and collected. Pierce BCA Assay Kit (Thermo Fisher) was utilized to detect exosome concentration. Nanoparticle tracking analysis was used for determining the profile of size distribution and measurement of concentration through a particle tracker (ParticleMetrix, Germany).

\section{Electron microscopy}

During roughly ten mins, nearly $50 \mu \mathrm{l}$ of exosome preparation was taken up by absorption in 300-mesh formvar/carbon coated grids. The grids were then dried at ambient temperature for half an hour and transferred to $3 \%$ phosphotungstic acid solution for negative staining. The exosomes were visualized by using an Olympus transmission electron microscope at $120.0 \mathrm{kV}$. Exosome images were taken using a CCD camera.

\section{Exosome uptake}

CV-MSC-derived exosomes were tagged by the PKH67 dye (Sigma-Aldrich) in accordance with the slightly modified protocol provided by the manufacturer. In brief, exosomes were subjected to suspension with $250 \mu \mathrm{L}$ PBS and blended with $750 \mu \mathrm{L}$ diluted PKH67 (1/50) (for cytomembrane labeling, in Diluent C), followed by $5 \mathrm{~min}$ of incubation at ambient temperature. After that, $2 \mathrm{~mL}$ BSA was used to end the labeling process. The isolation of labeled exosomes was performed as previously specified, followed by suspension in $1 \times$ PBS. 
In uptake experiments, labeled exosomes $(10 \mu \mathrm{g})$ were incubated with JAR cells seeded on 24-well plates at $37^{\circ} \mathrm{C}$ for $3 \mathrm{~h}$. The cells were rinsed and fixed by $4 \%$ paraformaldehyde in PBS for $30 \mathrm{~min}$ at ambient temperature. Nuclei staining was conducted with 4',6-diamidino-2-phenylindole (DAPI, Sigma).

\section{RNA-seq}

Samples were collected using TRIzol $(1 \mathrm{ml})$ (Thermo Fisher Scientific, and were placed at minus $80^{\circ} \mathrm{C}$. Libraries were prepared according to the instructions for an Illumina TruSeq RNA Sample Prep Kit, and sequencing was performed on a MiSeq instrument. Analysis of data (from Annoroad Gene Technology, Beijing, China) was performed on RSEM software. With labeling of no. SRR9943697-no. SRR9943702, all RNA-seq data are available at Sequence Read Archive (SRA).

\section{miRNA mimics and lentivirus infection}

Transient transfections for lentivirus infection were performed by Lipofectamine 2000 (Invitrogen, Carlsbad, USA). These transfected cells were extracted after being treated by puromycin to generate stable transfected cell lines.

The shRNA sequences are as follows: sh-Control, 5'- GCAAGCTGACCCTGAAGTT -3'; sh-TXNIP, 5'GCCTTCGGGTTCAGAAGAT-3'. Control microRNA, miR-135b-5p mimics were purchased from Hanbio (Shanghai, China). Control lentivirus (Vector), lentiviral constructs expressing full-length TXNIP, miR-135b$5 p$ overexpression and control lentiviruses (NC) were used to establish stably overexpressed cell lines.

\section{Protein isolation, western blot analysis and qRT-PCR}

Protein isolation, western blot analysis and qRT-PCR were carried out as previously described ${ }^{26}$.

The primary antibodies adopted for western blotting analysis are as follows: TXNIP (NBP2-75692, Novus, Cambridge, USA; 1/1000), CD9 (ab254175, Abcam, Cambridge, USA; 1/1000), Calnexin (ab22595, Abcam; 1/1000), $\beta$-catenin (\#8480, Cell Signaling Technology, Beverly, USA; 1:1000), GAPDH (ab181603, Abcam; 1:5000), Histone H3 (ab176842, Abcam; 1:1000), $\beta$-catenin (\#8480, Cell Signaling Technology, Beverly, USA; 1:1000).

The primer sequences are listed below: TXNIP, forward, 5'-TAGTGTAACCAGCGGCGTAT -3', reverse, 5'CACACCTCCACTATCACCCG-3'; GAPDH forward, 5'- AATGGGCAGCCGTTAGGAAA-3', reverse, 5'GCGCCCAATACGACCAAATC-3'. The primers for miR-135b-5p were purchased from Ribobio (Guangzhou, China).

\section{Cell proliferation analysis}

Cells $\left(5 \times 10^{3} /\right.$ well) were seeded in the 96 -well plates. The CCK-8 (Thermo Fisher Scientific, MA, USA) was added to evaluate cell viability each day as per the protocol. The OD value at $450 \mathrm{~nm}$ was detected through a microplate reader. Cell growth curves were carried out in triplicate. 


\section{Trophoblast invasion assay}

Two trophoblast lines $\left(5 \times 10^{5}\right.$ cells) were plated into transwell membrane (Corning, NY, USA). The pore membranes were covered by Matrige ${ }^{\mathrm{TM}}$ matrix dilution (1:10). The trophoblasts were then incubated for $12 \mathrm{~h}$. Medium (with 10\% FBS) treated with or without CV-MSCs-exosomes (control) was added into the lower layer. Transwell assays were conducted while trophoblasts were cultured without FBS for $24 \mathrm{~h}$. Subsequently, cell fixation was performed using $4 \%$ paraformaldehyde. Cells having traversed the membrane were quantified. Each experiment was conducted for at least 3 independent times.

\section{Luciferase reporter assays}

Cells $\left(3 \times 10^{3} /\right.$ well) were seeded in 96-well plates. The plasmids containing pGL3- TXNIP 3 ' UTR WT and pGL3- TXNIP 3' UTR MUT were procured from Hanbio (Shanghai, China). Co-transfection was performed with WT/MUT plasmids and miR-135b-5p mimic using Lipofectamine3000 (Thermo Fisher Scientific).

The reporter plasmids containing wild-type (TOPflash) or mutated (FOPflash) TCF/LEF DNA binding sites were purchased from Addgene (Addgene, Cambridge, USA) and Renilla reporter constructs were from Promega (Promega; Madison, USA). Luciferase reporters and Renilla reporters were co-transfected into cells with Lipofectamine 3000 (Thermo Fisher Scientific). Luciferase reporter activity was normalized against Renilla activity for $24 \mathrm{~h}$.

Dual-Luciferase Reporter Assay Kit (Promega) was used to detect luciferase activity.

\section{Bioinformatics analysis}

TargetScan (http://www.targetscan.org), a miRNA target predicting tool, was adopted for predicting the potential miRNA-miR135b-5p of TXNIP.

\section{Immunofluorescence}

Trophoblasts were treated with or without purified CV-MSCs-exosomes $(10 \mu \mathrm{g} / \mathrm{ml})$, and then cultured for $24 \mathrm{~h}$. They were then collected, separated and settled in $4 \%$ paraformaldehyde for $60 \mathrm{~min}$. The pellets were desected into $4-\mu \mathrm{m}$ thick sections and placed in paraffin. Later, the sections were bathed thrice in PBS, closed with $10 \%$ goat serum for $1 \mathrm{~h}$ and subsequently bathed twice in $0.2 \%$ Triton $\mathrm{X}-100$. Then, primary antibodies (TXNIP and $\beta$-catenin from Abcam, MA, USA), secondary antibodies (Invitrogen) and DAPI (Guangzhou RiboBio, Guangzhou, China) were used for cell incubation. And the images were take through a fluorescence microscope.

\section{Placental explant culture}

After being collected within 10 mins, all placentas were then processed in 30 min and observed carefully for abnormalities. Following thorough rinse in PBS thrice to remove remaining blood, the villous tissue was cut into small bits of tissue $(8 \mathrm{~mm} 3)$. DMEM/F12 (4 ml per well) supplemented with $1 \%$ 
penicillin/streptomycin and amphotericin B (Gibco, Carlsbad, CA) was adopted for culturing the placental explants in an incubator at $37^{\circ} \mathrm{C}$ at an oxygen concentration of $2 \%$ for 2 days. Following treatment with CV-MSC-exosomes for $24 \mathrm{~h}$, the explants were rinsed with PBS and then placed in liquid nitrogen.

\section{Statistical Analysis}

Two-tailed Student's t-test was adopted for data analysis. Each experiment was conducted independently in triplicate and all data were given as mean \pm SEM. Prism (Ver. 7) (GraphPad; La Jolla, USA) was used to analyze data. $P$ values less than 0.05 suggested statistical significance and were denoted by " $\star$ "; $P$ values

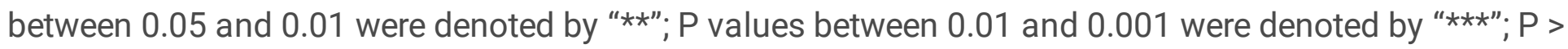
0.05 denoted no significance (n.s.).

\section{Results}

\section{Cell proliferation and invasion were promoted and TXNIP expression was inhibited in trophoblasts by CV-MSC- exosomes}

First, we performed the isolation of CV-MSCs from healthy placentas, and determined their multidirectional differentiation ability and surface marker expression (Fig. S1a-b). Then, we identified the exosomes isolated from CV-MSCs and observed the exosome endocytosis in trophoblasts (Fig. S2a-d). Next, we used the CCK-8 and transwell assays to examine the proliferation and invasion of trophoblasts treated with or without CV-MSC-derived exosomes. The trophoblasts treated with CV-MSC-derived exosomes displayed significantly higher proliferation rates and invasion abilities than those untreated cells (Fig. 1a-b, all $P<0.01$ ). To further verify the role of TXNIP in effects of CV-MSCs-exosomes on promoting trophoblasts proliferation and invasion, we examined the expression of TXNIP in placental explant cultures by immunohistochemical analysis. The CV-MSCs-exosome treated placental explants tissues exhibited significantly higher expression of Ki67 than the untreated explants, and the TXNIP expression was decreased after exosome treatment (Fig. 1C). Furthermore, to elucidate the possible molecular mechanisms of CV-MSC-derived exosomes, differentially expressed genes (DEGs) in exosometreated JAR cells were investigated using RNA sequencing, and the molecular mechanism of underlying functional changes in JAR was explored. We compared the transcriptomes between JAR cells co-cultured with or without CV-MSCs-exosomes for 48 h, and 2397 upregulated and 2014 downregulated genes were found to change more than two-fold (Fig. 1d). Based on the results, TXNIP was found to be downregulated significantly in JAR treated with CV-MSCs-derived exosomes, which was verified by qRTPCR and western blot assays (Fig. 1e-f).

These results indicated that the CV-MSCs-exosomes promoted trophoblast proliferation and invasion, and TXNIP was downregulated in this process. 


\section{Txnip Inhibited The Proliferation And Invasion Of Trophoblasts}

To better understand the role TXNIP played in trophoblasts after CV-MSC-derived exosome treatment, JAR cells were transfected with TXNIP shRNA and HTR-8/SVneoc cells were transfected with TXNIP overexpression lentiviruses. The mRNA levels of TXNIP were examined, and significant decrease and increase of TXNIP mRNA levels were respectively observed in transfected JAR and HTR-8/SVneoc cells (Fig. 2a). In consistence with the changes of mRNA levels, the TXNIP protein levels were also significantly changed in transfected trophoblasts compared to the control trophoblasts (Fig. 2b). Moreover, the growth of JAR cells was significantly promoted in the presence of TXNIP shRNA, while the TXNIP overexpression hindered HTR-8/SVneoc cells proliferation (Fig. 2c). In addition, we examined the invasion of transfected trophoblasts, and found that the cellular invasion changes in the corresponding trophoblasts shared the same curve as observed changes in proliferation (Fig. 2d). Our results suggested that TXNIP could be inhibited by CV-MSCs-exosomes which regulated the proliferation and invasion of trophoblasts.

\section{Txnip Suppressed $\beta$-catenin Pathway Activation In Trophoblasts}

Many researches have revealed the WNT/ $\beta$-catenin pathway contributes vital roles in intracellular metabolism regulation, suggesting that it is involved in TXNIP associated trophoblasts regulation ${ }^{27,28}$. Moreover, Gene Set Enrichment Analysis (GSEA) of trophoblasts revealed the WNT/ $\beta$-catenin pathway was among the most significantly upregulated pathways in trophoblasts treated with CV-MSCsexosomes (Fig. 3a). As a primary member in the typical WNT pathway, $\beta$-catenin is coordinated by a complex mesh of factors during WNT signal activation in the nucleus ${ }^{29}$. Therefore, we used TOP/FOP flash assays to investigate the effect of TXNIP on $\beta$-catenin and TCF-dependent transcriptional activity to identify the role of TXNIP in the typical WNT signaling. The TOP/FOP flash reporter activities were more significantly increased in shTXNIP JAR cells, than in the scrambled control cells. The reporter activity changes in TXNIP overexpressed HTR-8/SVneoc cells coincided with the change pattern of JAR cells (Fig. 3b). Moreover, the $\beta$-catenin and TXNIP expressions were evaluated by immunofluorescence and western blot. The relative $\beta$-catenin expression levels were significantly upregulated in the TXNIP-silenced JAR cells and downregulated in the TXNIP overexpressed HTR-8/SVneoc cells, compared to the scrambled control (Fig. 3c-d). The results suggested the possible involvement of TXNIP in modulating the $\beta$-catenin pathway by interacting directly with $\beta$-catenin in trophoblasts. Then, we investigated if the $\beta$ catenin pathway could be regulated by the TXNIP in trophoblasts.

\section{MiR-135b-5p was highly expressed in exosomes and targets TXNIP in trophoblast cells}

On the premise of above results, we sought to understand the upstream regulator of TXNIP in CV-MSCsexosomes. Since small non-coding RNAs could regulate expressions of genes and contribute critical roles 
in various process of physiological developmental, such as placental growth and function, potential regulatory miRNAs at the upstream of TXNIP were screened using bioinformatics tools. We selected miR135b for analysis over other miRNAs that might have interaction with TXNIP because miR-135b had been found to promote proliferation and invasion in many types of cancers ${ }^{30,31}$. The region of TXNIP bound with miR-135b-5p was found to be located in the TXNIP 3' UTR (Fig. 4a). Subsequently, RT-PCR assays revealed a higher miR-135b-5p level in CV-MSCs compared with CV-MSCs-exosomes (Fig. 4b). Furthermore, the miR-135b-5p level was significantly increased in trophoblasts treated with CV-MSCsexosomes in comparison with those treated with PBS (Fig. 4c).

To explore the role miR-135b-5p played in TXNIP regulation of trophoblasts, the trophoblasts were treated with miR-135b-5p mimics or control microRNA. The miR-135b-5p levels were then evaluated by RT-PCR assays, and high levels of miR-135b-5p were confirmed in JAR and HTR8/SVneo cells (Fig. 4d). Subsequently, luciferase assay was used to identify the interplay between the miR-135b and TXNIP $3^{\prime}$ UTR. The entire length of TXNIP 3' UTR was synthesized and was then inserted into a luciferase reporter plasmid, and miRNA mimics (miR-mimics) or scrambled negative control RNAs (miR-NC) were cotransfected. It revealed a significant reduction of luciferase activity in cells with miR-135b mimics (miRmimics) transfection, in comparison with those with control RNA transfection (Fig. 4e). In addition, the western blot assays confirmed the miR-135b-5p mimics-treated trophoblasts expressed higher levels of $\beta$ catenin protein and lower levels of TXNIP protein (Fig. 4f). These results indicate that miR-135b-5p may be delivered by CV-MSCs-exosomes and can directly regulate TXNIP expression in trophoblasts.

\section{Mir-135b-5p Advances Trophoblasts Proliferation And Invasion Via Txnip- $\beta$-catenin}

Next, to determine the role miR-135b-5p played in trophoblasts proliferation and invasion, lentiviral vector carrying miR-135b-5p were constructed and trophoblasts with stable miR-135b-5p expression were transfected with TXNIP-overexpression lentiviruses or vectors. Then the TXNIP overexpression was confirmed by the western blot (Fig. 5a). Furthermore, the luciferase activity raised by miR-135b increment was significantly decreased in cells transfected with TXNIP-overexpressed lentiviruses compared to cells transfected with vectors (Fig. 5b), indicating the effectiveness of our stably transfected constructs. Therefore, we examined the effect of TXNIP overexpression on miR-135b-mediated trophoblasts proliferation and invasion in vitro. The proliferation and invasion of trophoblasts were then determined by CCK-8 and transwell assays. The miR-135b-5p overexpression markedly promoted proliferation and invasion of trophoblasts, which could be attenuated by TXNIP overexpression (Fig. 5c-d). These data confirmed that miR-135b-5p promoted trophoblasts proliferation and invasion through TXNIP inhibition.

To summarize, these data indicate that CV-MSC-derived exosomes may inhibit the TXNIP- $\beta$-catenin pathway through miR-135b-5p.

\section{Discussion}


The placenta maintains its functions mainly through trophoblasts. Dysfunctional trophoblast invasion can ultimately cause the $\mathrm{PE}$, a great obstetric syndrome. MSCs are progenitor cells with capability if selfrenewal, and numerous studies have revealed the proangiogenic potentiality of MSCs deriving from their regenerating and paracrine activities. MSC-derived treatment turns up as one of most promising therapies for ischemic diseases. Moreover, it has recently been revealed that the therapeutic effects of MSC-derived exosomes on ischemic cardiac disease, renal impairment, and wound are analogous to the functional effect of their parental cells, indicating active roles of MSC-derived exosomes. Nevertheless, the underlying mechanisms in MSC-derived exosomes mediating these therapeutic effects remain unclear. In this study, we evaluated the roles of foetal CV-MSCs in the proliferative and invasive capacity of trophoblasts, and provide evidences that CV-MSC-derived exosomes mediate inhibition of TXNIP expression and $\beta$-catenin pathway, which is also regulated by the miR-135b-5p in exosomes.

Exosomes, containing various subtypes, both participate in regular biological processes and in pathological disorders. Notwithstanding that much remains unknown regarding their functions, more and more studies have revealed that exosomes function as crucial intercellular message transmitters. Exosomes can directly interact with their target cells, which accounts for exosomes' involvement in mediating intercellular signaling. The capability for packaging and transporting miRNAs enables exosomes to participate in intercellular communication. Exosomes are carriers of various miRNAs, are bound to particular targeted cytomembrane receptors, and participate in certain regulatory pathways to regulate diverse physiological and pathological processes. Recently, effects of exosomes on immunotherapy have increasingly been uncovered.

Many researches have demonstrated miRNAs show differential expression between normal placentas and placentas of PE patients, suggesting a significant role by miRNAs in physiological processes in placentas. Despite limited information on miRNA regulating functional development in placentas, multiple studies indicate that PE-related differentially expressed miRNAs contribute vital roles in the regulation of placental development and function through targeting functional genes. It is known that miRNA inhibits protein expression mainly through inhibiting translation. The miRNA carried by CV-MSCsexosomes could be an upstream regulator of trophoblast function. Shunsuke et al. found that miR-135b contributed vital roles in regulating the trophoblast invasion in hypoxic conditions through direct regulation of CXCL12 expression ${ }^{32}$. Meanwhile, miR-135b was also identified as being abnormally expressed in PE pregnancies, indicating its possible involvement in PE pathology ${ }^{33}$. In our study, this wellknown tumor-associated microRNA was found to interact with TXNIP and overexpressed in CV-MSCsexosomes, implying an intersection between CV-MSCs-exosomes and TXNIP in trophoblasts through miR135b-5p.

TXNIP has been reported to be capable of binding to and activating the NLR family pyrin domain containing 3 (NLRP3) inflammasome, and contribute a key role in immuno-inflammatory modulation. TXNIP is significantly increased in oxygen-deficient trophoblasts, which can be noticed in placental tissue from early-onset PE pregnancies. Moreover, abnormal activation of $\mathrm{Wnt} / \beta$-catenin may contribute to the development of PE by dysregulating the invasion and proliferation of trophoblast ${ }^{34}$. In our CV-MSCs- 
exosomes treated trophoblasts RNA-seq analysis, TXNIP and WNT/ $\beta$-catenin pathways were substantially downregulated. Based on these findings, we propose that TXNIP/ $\beta$-catenin pathways could be massively involved in mediating the inflammatory dysregulation in PE, which could be reversed by CVMSCs-exosomes miRNA.

\section{Conclusion}

Our study revealed that miR-135b-5p carried by CV-MSCs derived-exosomes promoted the trophoblast proliferation and invasion in vitro, partially due to inactivation of $\beta$-catenin pathways induced by the TXNIP downregulation. However, it remains undetermined how CV-MSCs affect other placenta-based cells in vitro or even in vivo; therefore, further studies are needed on whether CV-MSCs have similar effects on them. More studies with respect to how miR-315b-5p regulates TXNIP and $\beta$-catenin pathways will be important for a thorough understanding of the PE pathogenesis.

\section{Abbreviations}

CV-MSCs

Chorionic villous mesenchymal stem cells; PE:preeclampsia; MSCs:Mesenchymal stem cells; TXNIP:Thioredoxin-interacting protein; ASK-1:Apoptosis signal-regulating kinase-1; ROS:Reactive oxygen species; SCCM:Stem cell culture medium; NC:control lentiviruses; PBS:Phosphate-buffered saline; FBS:Foetal bovine serum; NLRP3:NLR family pyrin domain containing 3.

\section{Declarations}

\section{Ethics approval and consent to participate}

The study was approved by the Ethics Committee of the Qingdao University Affiliated Hospital. All participants provided a written informed consent.

\section{Consent for publication}

Not applicable.

\section{Availability of supporting data}

The datasets used and/or analyzed during the current study are available from the corresponding author on reasonable request.

\section{Competing interests}

The authors declare no potential conflicts of interest.

\section{Funding}


This study was supported by the Clinical Medicine+X Project of the Affiliated Hospital of Qingdao University.

\section{Authors' contributions}

Conceived and designed the experiments: YC, YW and YY. Performed the experiments: YW, YC, YZ, GG and JZ. Collected samples: YL, NZ, JL, LL, HY and PT. Analyzed the data: YC, XW, KY, CL, HZ and ML. Wrote the paper: $\mathrm{MZ}, \mathrm{XH}$ and $\mathrm{YC}$. All authors read and approved the final manuscript.

\section{Acknowledgements}

No funders had no role in the study design, in the collection, analysis, and interpretation of the data or in writing the manuscript. We thank the Department of Obstetrics, the Affiliated Hospital of Qingdao University, Qingdao, China, for providing tissue samples.

\section{References}

1. Shah S, Gupta A. Hypertensive Disorders of Pregnancy. Cardiol Clin. 2019;37:345-54. doi:10.1016/j.ccl.2019.04.008.

2. Turco MY, Moffett A. Development of the human placenta. Development 146, doi:10.1242/dev.163428 (2019).

3. Ridder A, Giorgione V, Khalil A, Thilaganathan B, Preeclampsia. The Relationship between Uterine Artery Blood Flow and Trophoblast Function. Int J Mol Sci 20, doi:10.3390/ijms20133263 (2019).

4. Rana S, Lemoine E, Granger JP, Karumanchi SA, Preeclampsia. Pathophysiology, Challenges, and Perspectives. Circulation research. 2019;124:1094-112. doi:10.1161/CIRCRESAHA.118.313276.

5. Pollheimer J, Vondra S, Baltayeva J, Beristain AG, Knofler M. Regulation of Placental Extravillous Trophoblasts by the Maternal Uterine Environment. Frontiers in immunology. 2018;9:2597. doi:10.3389/fimmu.2018.02597.

6. MacDonald ES, Barrett JG. The Potential of Mesenchymal Stem Cells to Treat Systemic Inflammation in Horses. Front Vet Sci. 2019;6:507. doi:10.3389/fvets.2019.00507.

7. Heazlewood CF, et al. High incidence of contaminating maternal cell overgrowth in human placental mesenchymal stem/stromal cell cultures: a systematic review. Stem cells translational medicine. 2014;3:1305-11. doi:10.5966/sctm.2014-0051.

8. Abumaree $\mathrm{MH}$, et al. Phenotypic and Functional Characterization of Mesenchymal Stem Cells from Chorionic Villi of Human Term Placenta. Stem Cell Reviews Reports. 2012;9:16-31. doi:10.1007/s12015-012-9385-4.

9. Wang Z, et al. Transplantation of human villous trophoblasts preserves cardiac function in mice with acute myocardial infarction. J Cell Mol Med. 2017;21:2432-40. doi:10.1111/jcmm.13165.

10. Paulraj B, Kannaiyan J, Muthukutty P, Iqbal MDT. Villous chorion: A potential source for pluripotentlike stromal cells. Journal of Natural Science Biology Medicine 8, doi:10.4103/0976-9668.210011 
(2017).

11. Farooqi $A A$, et al. Exosome biogenesis, bioactivities and functions as new delivery systems of natural compounds. Biotechnol Adv. 2018;36:328-34. doi:10.1016/j.biotechadv.2017.12.010.

12. Cheng N, et al. Recent Advances in Biosensors for Detecting Cancer-Derived Exosomes. Trends in biotechnology. 2019;37:1236-54. doi:10.1016/j.tibtech.2019.04.008.

13. Wang L, Zhang L. Circulating Exosomal miRNA as Diagnostic Biomarkers of Neurodegenerative Diseases. Front Mol Neurosci. 2020;13:53. doi:10.3389/fnmol.2020.00053.

14. Chen DB, Wang W. Human placental microRNAs and preeclampsia. Biol Reprod. 2013;88:130. doi:10.1095/biolreprod.113.107805.

15. Awamleh Z, Gloor GB, Han VKM. Placental microRNAs in pregnancies with early onset intrauterine growth restriction and preeclampsia: potential impact on gene expression and pathophysiology. BMC Med Genomics. 2019;12:91. doi:10.1186/s12920-019-0548-x.

16. Lv Y, et al. Roles of microRNAs in preeclampsia. J Cell Physiol. 2019;234:1052-61. doi:10.1002/jcp.27291.

17. Mouillet JF, Chu T, Nelson DM, Mishima T, Sadovsky Y. MiR-205 silences MED1 in hypoxic primary human trophoblasts. FASEB journal: official publication of the Federation of American Societies for Experimental Biology. 2010;24:2030-9. doi:10.1096/fj.09-149724.

18. Sullivan WJ, et al. Extracellular Matrix Remodeling Regulates Glucose Metabolism through TXNIP Destabilization. Cel/ 175, 117-132 e121, doi:10.1016/j.cell.2018.08.017 (2018).

19. Kumar A, Mittal R, Mapping Txnip. Key connexions in progression of diabetic nephropathy. Pharmacological Reports. 2018;70:614-22. doi:10.1016/j.pharep.2017.12.008.

20. Nasoohi S, Ismael S, Ishrat T. Thioredoxin-Interacting Protein (TXNIP) in Cerebrovascular and Neurodegenerative Diseases: Regulation and Implication. Mol Neurobiol. 2018;55:7900-20. doi:10.1007/s12035-018-0917-z.

21. Alhawiti NM, Mahri A, Aziz S, Malik MA, S. S. \& Mohammad S. TXNIP in Metabolic Regulation: Physiological Role and Therapeutic Outlook. Curr Drug Targets. 2017;18:1095-103. doi:10.2174/1389450118666170130145514.

22. Yoshihara E, et al. Thioredoxin/Txnip: Redoxisome, as a Redox Switch for the Pathogenesis of Diseases. Frontiers in immunology 4, doi:10.3389/fimmu.2013.00514 (2014).

23. Coucha M, Shanab AY, Sayed M, Vazdarjanova A, El-Remessy AB. Modulating Expression of Thioredoxin Interacting Protein (TXNIP) Prevents Secondary Damage and Preserves Visual Function in a Mouse Model of Ischemia/Reperfusion. Int J Mol Sci 20, doi:10.3390/ijms20163969 (2019).

24. Heo MJ, et al. Alcohol dysregulates miR-148a in hepatocytes through Fox01, facilitating pyroptosis via TXNIP overexpression. Gut. 2019;68:708-20. doi:10.1136/gutjnl-2017-315123.

25. Yu L, et al. Melatonin rescues cardiac thioredoxin system during ischemia-reperfusion injury in acute hyperglycemic state by restoring Notch1/Hes1/Akt signaling in a membrane receptor-dependent manner. J Pineal Res 62, doi:10.1111/jpi.12375 (2017). 
26. Wang $Y$, et al. Epidural adipose tissue-derived mesenchymal stem cell activation induced by lung cancer cells promotes malignancy and EMT of lung cancer. Stem Cell Res Ther. 2019;10:168. doi:10.1186/s13287-019-1280-3.

27. Schaefer KN, Peifer M. Wnt/Beta-Catenin Signaling Regulation and a Role for Biomolecular Condensates. Dev Cell. 2019;48:429-44. doi:10.1016/j.devcel.2019.01.025.

28. Nusse R, Clevers H. Wnt/beta-Catenin Signaling, Disease, and Emerging Therapeutic Modalities. Cell. 2017;169:985-99. doi:10.1016/j.cell.2017.05.016.

29. Pai SG, et al. Wnt/beta-catenin pathway: modulating anticancer immune response. J Hematol Oncol. 2017;10:101. doi:10.1186/s13045-017-0471-6.

30. Hu Y, Wang Q, Zhu XH. MiR-135b is a novel oncogenic factor in cutaneous melanoma by targeting LATS2. Melanoma Res. 2019;29:119-25. doi:10.1097/CMR.0000000000000524.

31. Bai M, et al. miR-135b Delivered by Gastric Tumor Exosomes Inhibits FOX01 Expression in Endothelial Cells and Promotes Angiogenesis. Molecular therapy: the journal of the American Society of Gene Therapy. 2019;27:1772-83. doi:10.1016/j.ymthe.2019.06.018.

32. Tamaru S, Tochigi MY, Kajihara H, Okazaki T, Okagaki Y, Kamei R, Ishihara Y, Itakura O. A. MicroRNA135b suppresses extravillous trophoblast-derived. HTR-8/SVneo cell invasion by directly down regulating CXCL12. under low oxygen conditions. Biochem Biophys Res Commun. 2015;461:421-6. doi:10.1016/j.bbrc.2015.04.055.

33. Yang S, Li H, Ge Q, Guo LI, Chen F. Deregulated microRNA species in the plasma and placenta of patients with preeclampsia. Mol Med Rep. 2015;12:527-34. doi:10.3892/mmr.2015.3414.

34. Wang X, Zeng ZZ, Wang X, Zhang J, Song L, Shi W. Y Wnt/ $\beta$-catenin signaling pathway in severe preeclampsia. J Mol Histol. 2018;49:317-27. doi:10.1007/s10735-018-9770-7.

\section{Figures}


a

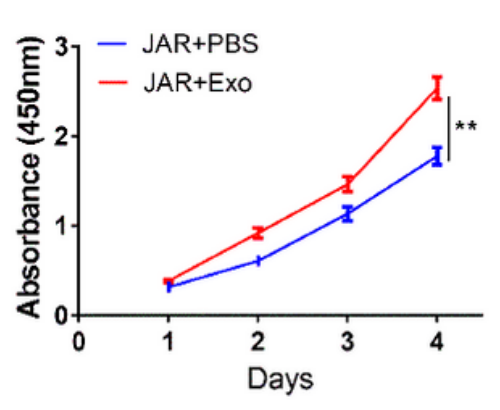

b

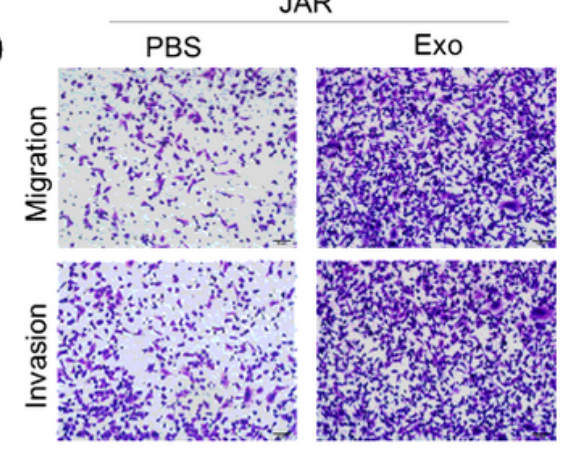

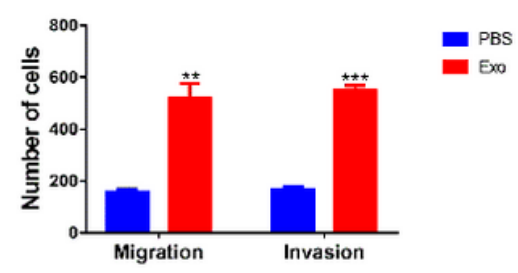

$\mathrm{IHC}$

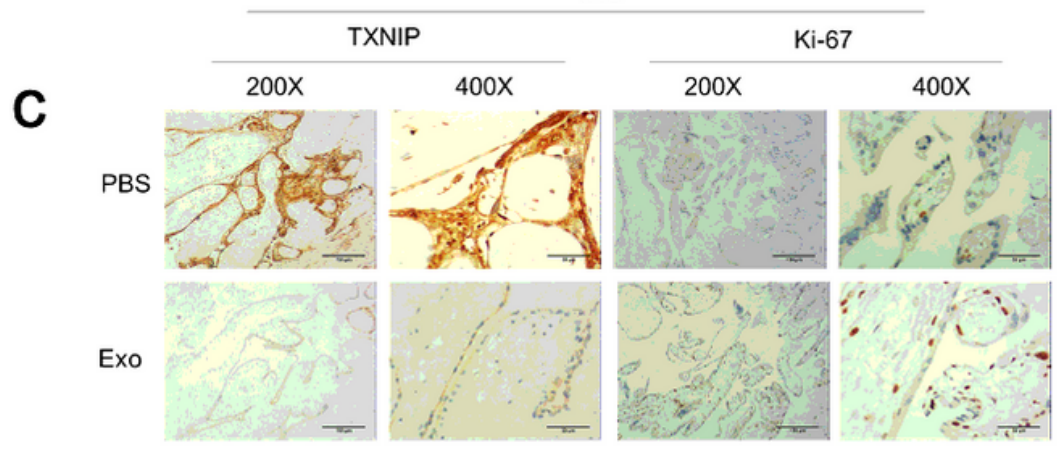

e

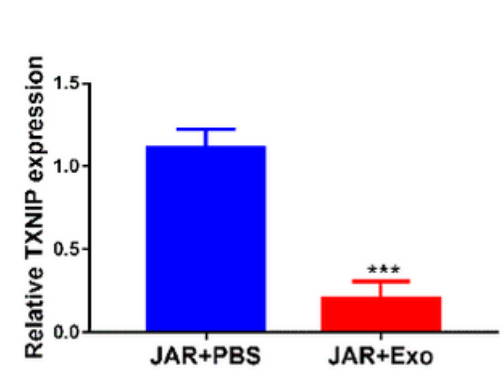

f
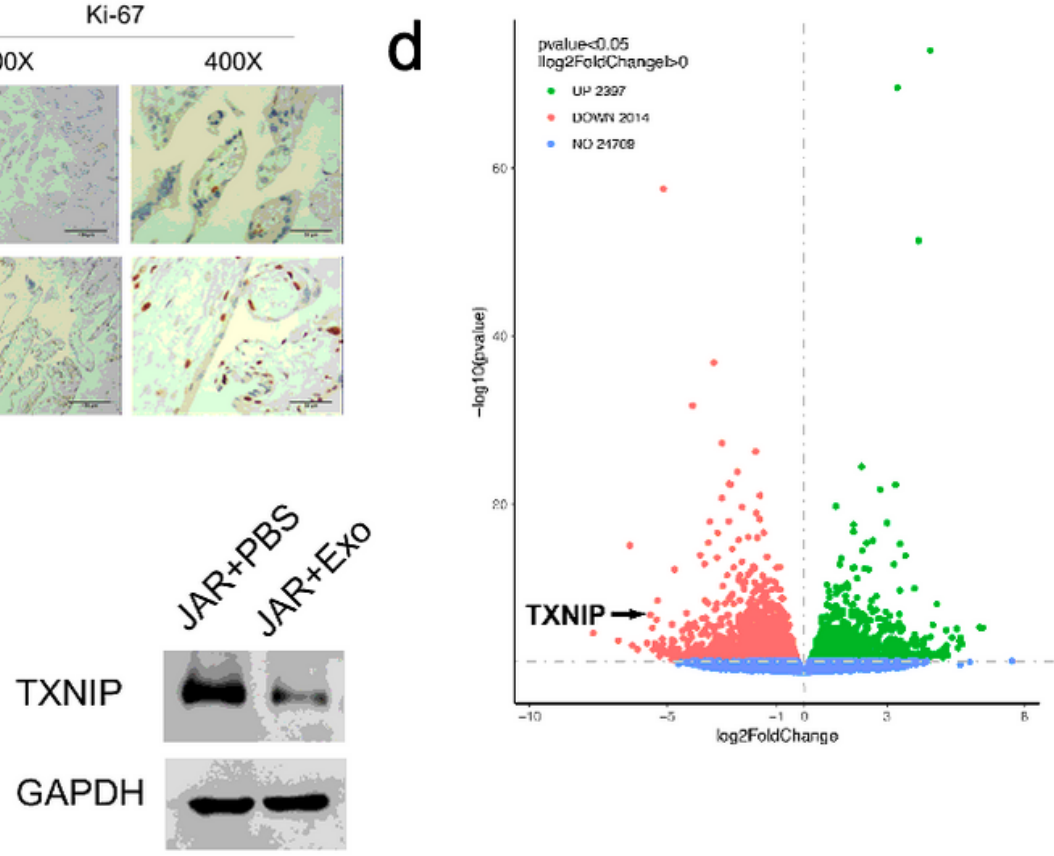

\section{Figure 1}

CV-MSC-derived exosomes inhibit TXNIP expression, promote cell proliferation and invasion of trophoblasts. (a) CCK8 assays from JAR cells treated with PBS or CV-MSCs-derived exosomes to evaluate cell proliferation. (b) Representative images and graphic analysis of transwell assays from JAR cells. (c) TXNIP and Ki67 expression in placental explants treated with exosomes or PBS were examined by IHC. (d) Heatmap of differential mRNA expression between JAR+PBS and JAR+Exo. (e) qRT-PCR analysis of TXNIP from JAR+PBS and JAR+Exo groups. (f) Western blot analysis of TXNIP from indicated cells. 
a

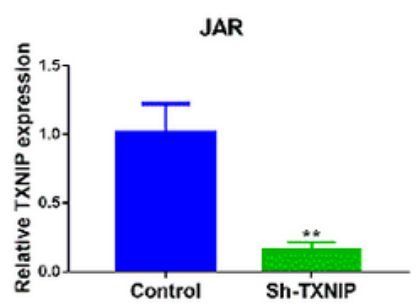

C

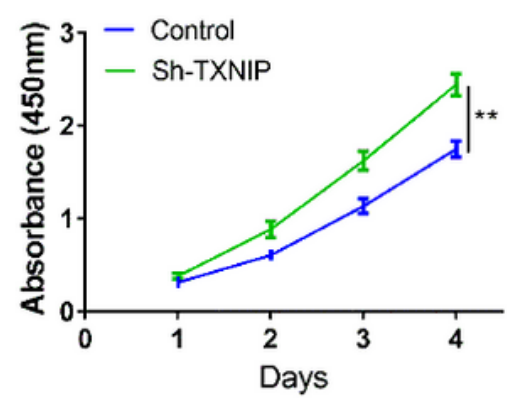

d

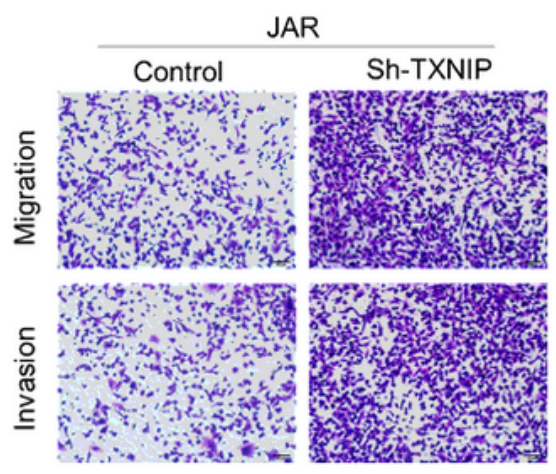

JAR

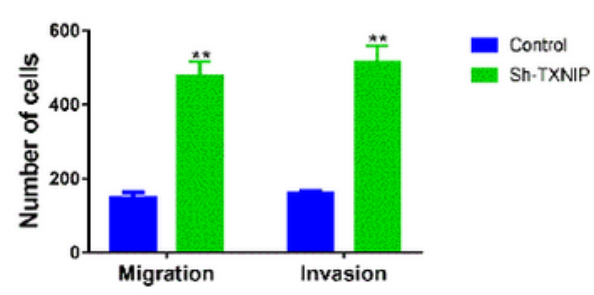

b

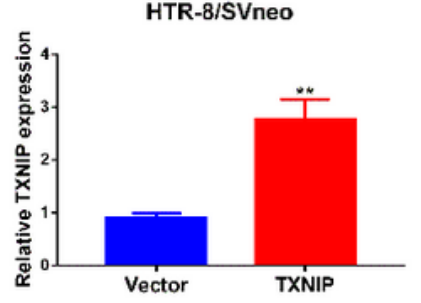

TXNIP

GAPDH
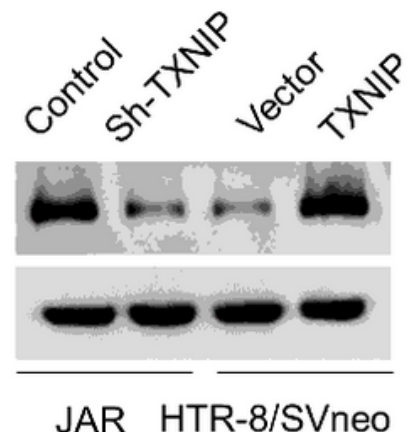

HTR-8/SVneo
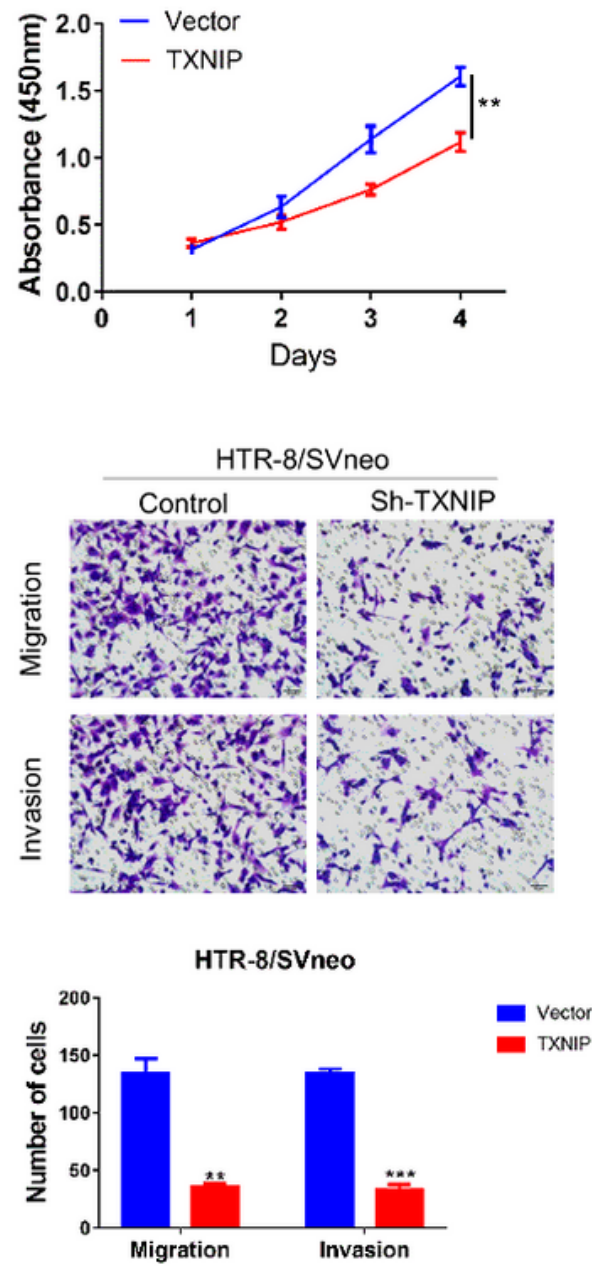

\section{Figure 2}

Loss of TXNIP enhances proliferation and invasion of trophoblasts in vitro and in vivo. (a-b) qRT-PCR and western blot analysis of TXNIP to evaluate knockdown/overexpression efficiency of lentiviruses. (c) Cell proliferation was evaluated by CCK8 assays in indicated groups. (d) Graphic analysis of transwell assays. 


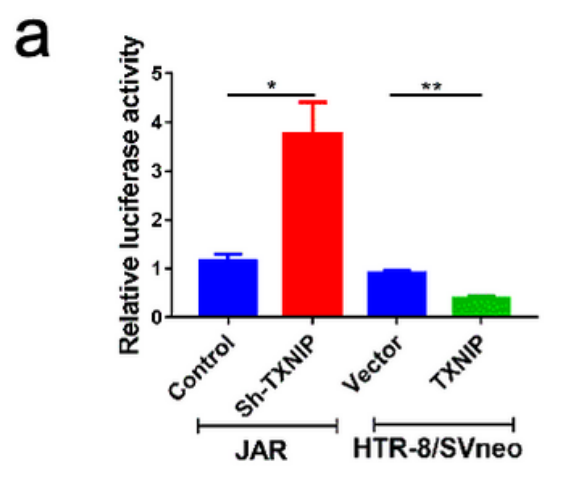

C

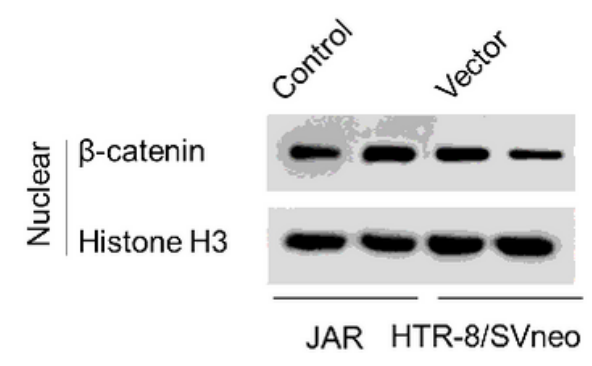

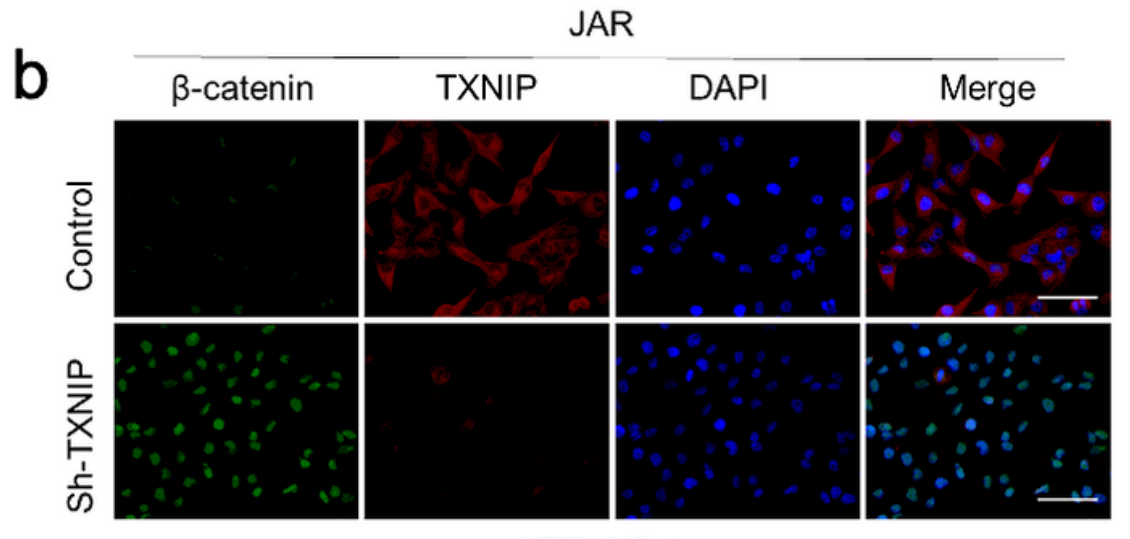

HTR-8/SVneo

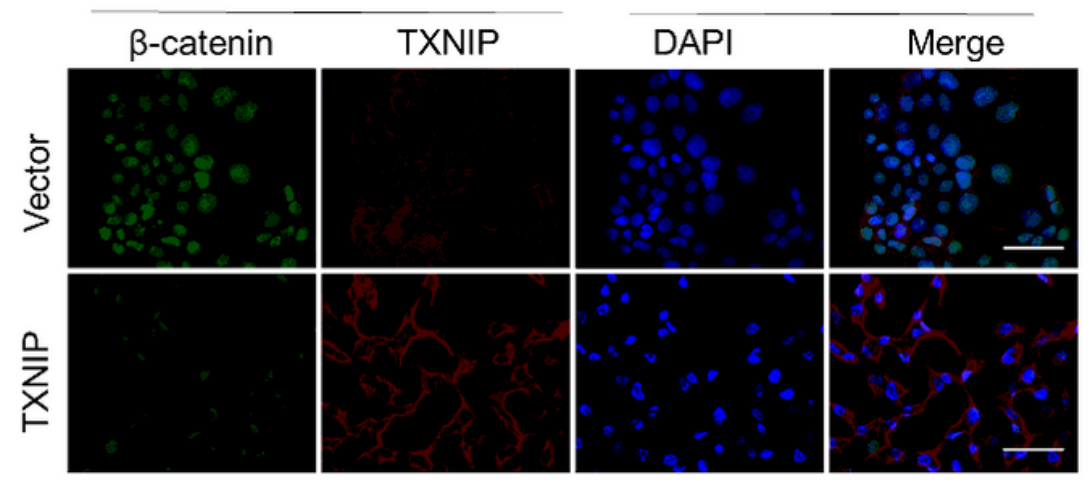

\section{Figure 3}

TXNIP represses the activation of $\beta$-catenin pathway in trophoblasts. (a) Graphic representation of relative levels of TOP/FOP luciferase activity in JAR cells with TXNIP knockdown or HTR-8/SVneoc cells with TXNIP overexpression. (b) Representative images of co-localization of TXNIP (red) with $\beta$-catenin (green) in JAR cells or HTR-8/SVneoc cells revealed by fluorescent microscopy. Scale bar $=25 \mu \mathrm{m}$. (c) Western blot analysis of $\beta$-catenin protein levels in nucleus isolated from indicated stably transfected two cells. Histone H3 served as the loading control. 
a

TXNIP 3' UTR - WT $\quad$ 5'... CACCAAAGGUCUUAAAAGCCAUU...3'

miR-135b-5p

TXNIP 3' UTR - MUT 3'AGUGUAUCCUUACUUUUCGGUAU 5'

5'... CACCAAAGgucuUAAAGTAAGUU...3'

C

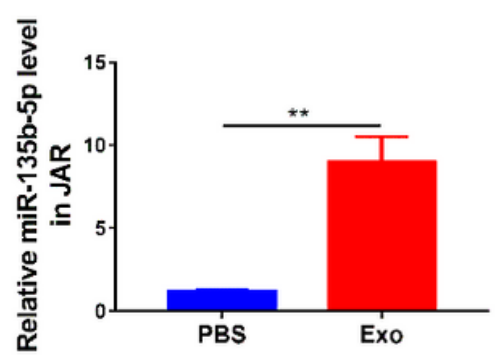

e

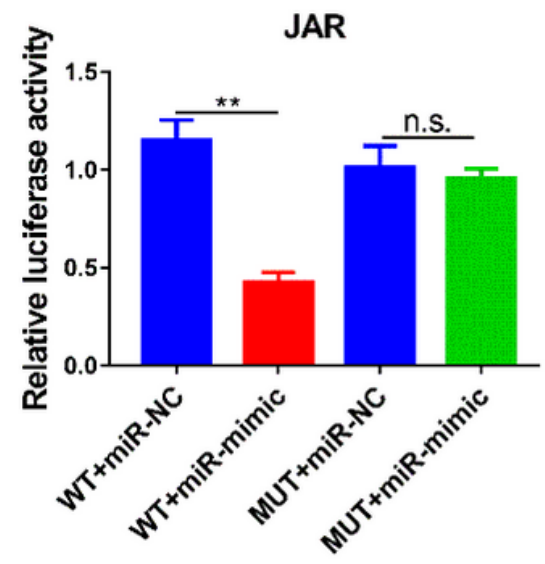

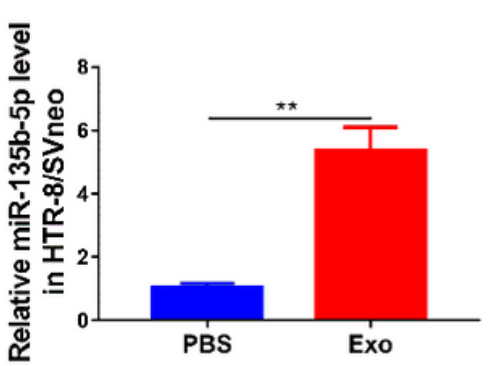

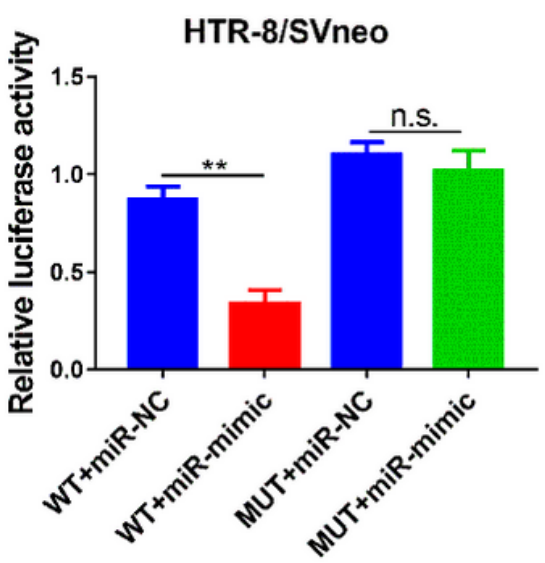

b

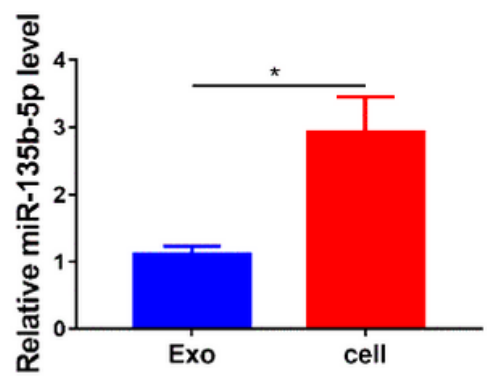

d

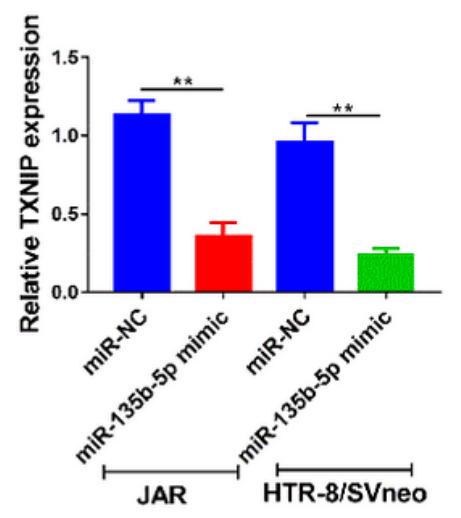

f

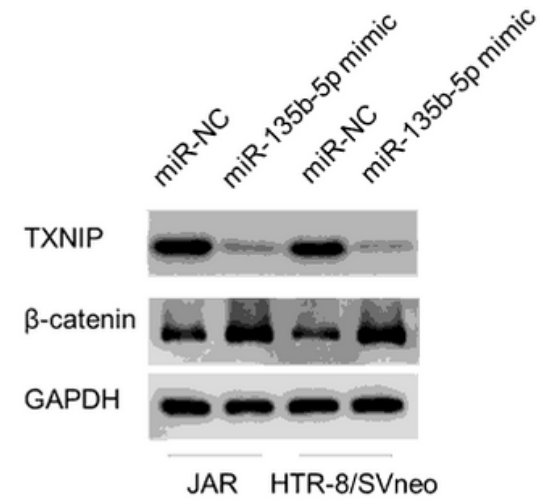

Figure 4

miR-135b-5p is highly expressed in exosomes and targets TXNIP in trophoblast cells. (a) Schematic diagram of miR-135b-5p and its putative binding sequence in the wild-type (WT) and mutant (MUT) 3'UTR of TXNIP. (b) qRT-PCR analysis of miR-135b-5p levels in CV-MSCs-derived exosomes and CV-MSCs. (c) qRT-PCR analysis of miR-135b-5p levels JAR cells and HTR-8/SVneoc cells treated with PBS or exosomes. (d) qRT-PCR analysis of TXNIP mRNA expression in cells treated miR-135b-5p mimic or control microRNA. (e) Relative luciferase activity in indicated cell groups. (f) Western blot analysis of TXNIP and $\beta$-catenin in JAR and HTR-8/SVneoc cells treated miR-135b-5p mimic or control microRNA. 


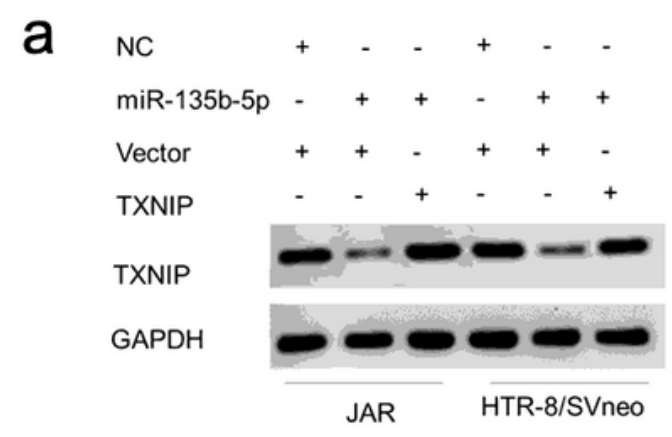

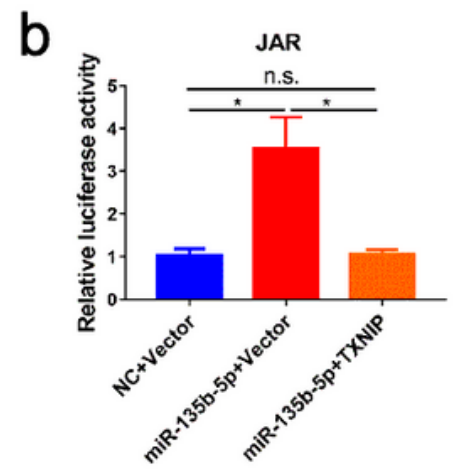

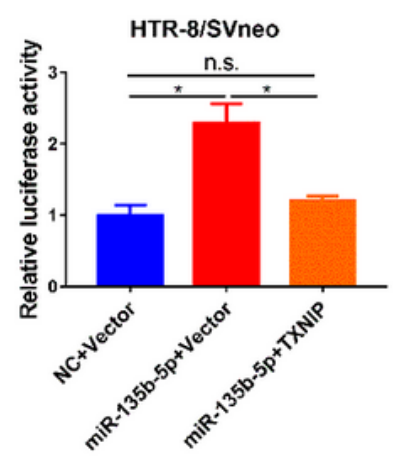

C

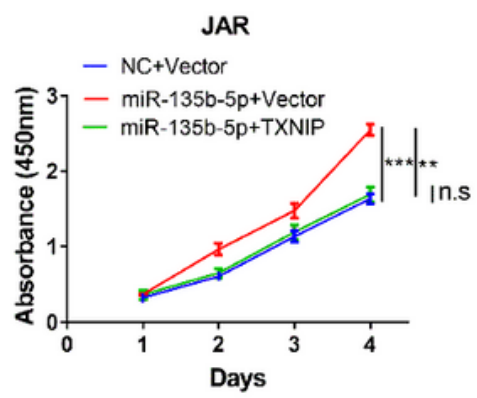

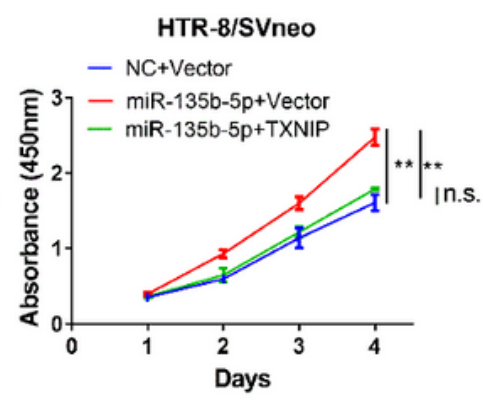

d
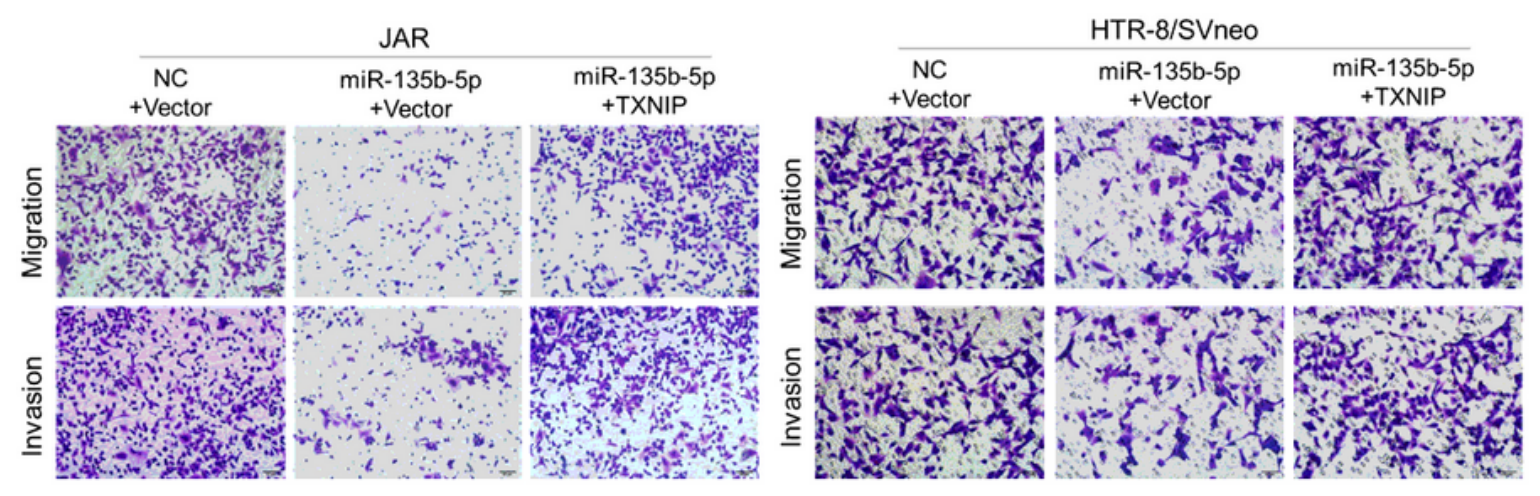

JAR

HTR-8/SVneo
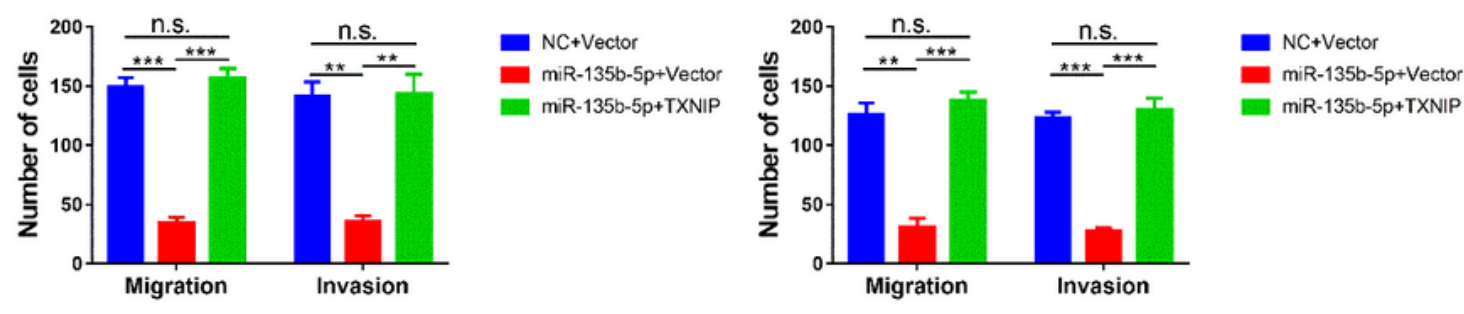

\section{Figure 5}

miR-135b-5p promotes cell proliferation, migration and invasion via TXNIP- $\beta$-catenin. (a) Western blot analysis of TXNIP expression from stably transfected cells to evaluate efficiency of lentiviruses. (b) Relative levels of TOP/FOP luciferase activity in indicated groups. Renilla activity was used to normalize luciferase reporter activity. (c) CCK8 assays to evaluate cell proliferation. (d) Representative images and graphic analysis of transwell assays from JAR and HTR-8/SVneoc cells. 


\section{Supplementary Files}

This is a list of supplementary files associated with this preprint. Click to download.

- Onlinefloatimage6.png

- Onlinefloatimage7.png 